\title{
Oscillatory Patterns in the Amount of Demand for Dental Visits: An Agent Based Modeling Approach
}

\author{
Maryam Sadeghipour ${ }^{1}$, Peyman Shariatpanahi $^{2}$, Afshin Jafari ${ }^{3}$, Mo- \\ hammad Hossein Khosnevisan ${ }^{1,4}$, Arezoo Ebn Ahmady \\ 1Department of Community Oral Health, Dental School, Shahid Beheshti University of Medical Sciences, \\ Daneshjoo Ave, Velenjak, Iran \\ ${ }^{2}$ Institute of Biochemistry and Biophysics, University of Tehran, Enghelab Square, Tehran, 13145-1384 Iran \\ ${ }^{3}$ Faculty of Management, University of Tehran, Amir Abad, North Kargar Street, Tehran, Iran \\ ${ }^{4}$ Preventive Dentistry Research Center, Research Institute of Dental Sciences, Shahid Beheshti University of \\ Medical Sciences, Tehran, Iran. \\ Correspondence should be addressed to pshariatpanahi@ut.ac.ir
}

Journal of Artificial Societies and Social Simulation 19(3) 10, 2016

Doi: 10.18564/jasss.3124 Url: http://jasss.soc.surrey.ac.uk/19/3/10.html

Received: 13-10-2015 Accepted: 16-05-2016 Published: 30-06-2016

\begin{abstract}
There are some empirical evidences indicating that there is a collective complex oscillatory pattern in the amount of demand for dental visit at society level. In order to find the source of the complex cyclic behavior, we develop an agent-based model of collective behavior of routine dental check-ups in a social network. Simulation results show that demand for routine dental check-ups can follow an oscillatory pattern and the pattern's characteristics are highly dependent upon the structure of the social network of potential patients, the population, and the number of effective contacts between individuals. Such a cyclic pattern has public health consequences for patients and economic consequences for providers. The amplitude of oscillations was analyzed under different scenarios and for different network topologies. This allows us to postulate a simulation-based theory for the likelihood observing and the magnitude of a cyclic demand. Results show in case of random networks, as the number of contacts increases, the oscillatory pattern reaches its maximum intensity, for any population size. In case of ringing lattice networks, the amplitude of oscillations reduces considerably, when compared to random networks, and the oscillation intensity is strongly dependent on population. The results for small world networks is a combination of random and ring lattice networks. In addition, the simulation results are compared to empirical data from Google Trends for oral health related search queries in different United States cities. The empirical data indicates an oscillatory behavior for the level of attention to dental and oral health care issues. Furthermore, the oscillation amplitude is correlated with town's population. The data fits the case of random networks when the number of effective contacts is about 4-5 for each person. These results suggest that our model can be used for a fraction of people deeply involved in Internet activities like Web-based social networks and Google search.
\end{abstract}

Keywords: Dental Health Care, Dental Routine Visit, Oscillatory Patterns, Agent Based Modeling, Google Trends

\section{Introduction}

1.1 Periodic health check-ups are critical in public health and provide an effective preventive measure that can mitigate chronic illnesses through early detection. With routine check-ups, early stages treatments can promote health and quality of life effectively; although, most people do not comply with such recommended check-ups. One obvious example is dental check-up.

1.2 Routine dental visits are critical for dental health promotion. Epidemiologic evidence shows that people who have regular dental visits have better oral health conditions than others (Catteau et al. 2012; Hawley et al. 1997; Catteau et al. 2014. Sheiham et al. 1985). The effects of routine dental visit on reducing level of dental caries is well known (Vehkalahti \& Paunio 1988). For example, a cohort study in New Zealand reported that people who 


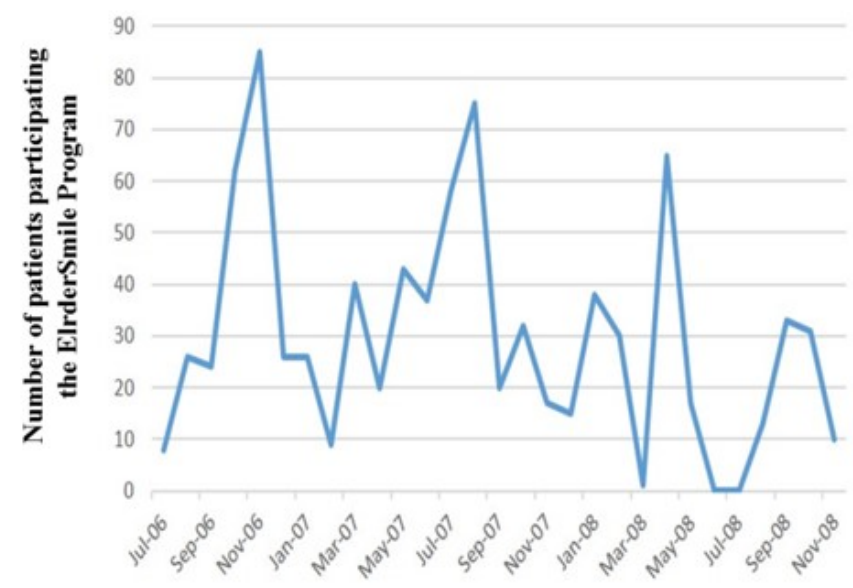

Figure 1: Oscillatory behavior of participants in the dental routine visit of the ElderSmile preventive screening program (Marshall et al.2009: Metcalf et al.2013).

do not maintain regular visits have three times higher levels of dental caries (Gilbert et al. 1997). It was demonstrated in a UK study that, self-reported quality of life was considerably higher for people who regularly go for routine dental visits (Mc Grath \& Bedi 2001). Positive effects of routine dental care have also been observed in health care behaviors such as tooth brushing (Casanova-Rosado et al. 2014) as well as in general health literacy (Wiener \& Shockey 2014). Although proper frequency of routine dental visits can be different for different individuals and societies, on average, presumably six months period may be the optimal interval between two successive visits as advised by most dentists (Frame et al. 2000).

1.3 Since promoting routine dental visit is an important concern for health practitioners, understanding factors that promote such visits is crucial. Previous studies have shown that lack of compliance with routine dental visits may be rooted in economic reasons (Peltzer \& Pengpid 2014), stress and anxieties associated with visiting dentists (Sohn \& Ismail 2005), oral health knowledge (Wiener \& Shockey|2014) and perceived oral health status Sohn \& Ismail 2005). Although there is a rich literature investigating individual factors, no study has examined how these individual level factors can influence public behavior in aggregate.

1.4 Many examples exist on how individuals can influence each other's health seeking behavior. Family members can have an impact. If parents start teaching their toddlers to brush their teeth in early ages, their other healthrelated preventative activities can influence their children's long term healthy behavior and improve their routine habits. Similarly, friends and colleagues can influence healthy behaviors. Such social influences in aggregate can lead to counter-intuitive emerging behaviors, i.e., a characteristic of complex social systems.

1.5 A series of recent studies by Metcalf et al. (2013) showed that dental visits, in aggregate, can follow wild oscillatory patterns. Specifically, they showed that dental visit promotion programs in New York (the ElderSmile program) have been going through cyclic patterns of demand. They argued that the oscillatory high level aggregate patterns were complex behaviors and thus difficult to explain with linear regression models (Marshall et al. 2009. Metcalf et al. 2013. Figure 1 shows the observations from the ElderSmile preventive screening program in New York City by the Columbia University College of Dental Medicine during July-2006 to November-2008 Metcalf et al. 2013). This program operated in North Manhattan (in which the elderly population have many friendship connections with their neighbors) and the trend of the participating population showed an oscillatory behavior with an average period of about 8 months. It should be noted that the pattern is not seasonal. Therefore, it was suggested that the pattern should be described by modeling the interactions among elders through their friendship network. This interaction somehow synchronizes the friends' decision to go for the visit (Metcalf et al.|2013). It should also be mentioned that this oscillatory pattern potentially might have serious implications on resources for the screening program as well as other similar programs.

1.6 By analyzing the big data provided by Google Trends service, we find another evidence of potential complex and cyclic patterns in people's attention to dental health. Google query trends for the word "dentist" in different US towns (Figure 2 show that public attention, especially in smaller towns, has been following oscillatory patterns. As Fig. 2 demonstrates, in a sample of three small and three large US towns, public attention to dental care represented by their online behavior, follows a cyclic patterns. The highest attention (search) points for different towns happen in various months, showing the patterns are not limited to seasonal changes. The time period of these patterns are different between 4-7 months. In addition, the difference between oscillation amplitudes in 


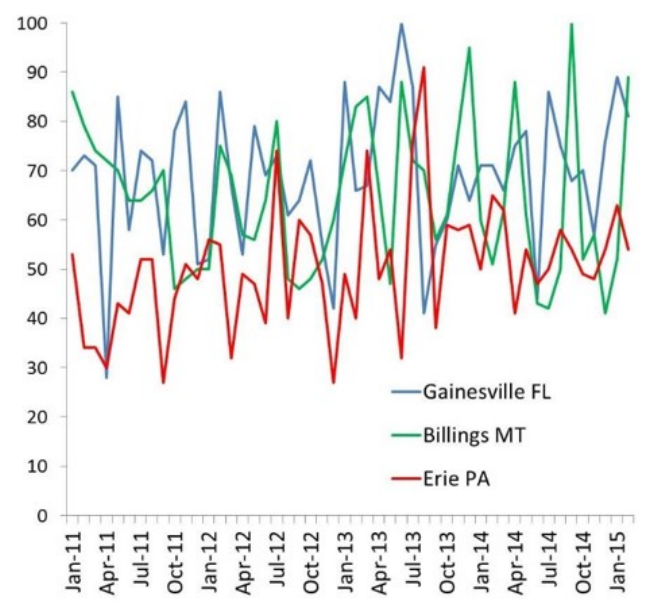

(a) three small towns

(populations around 100,000 )

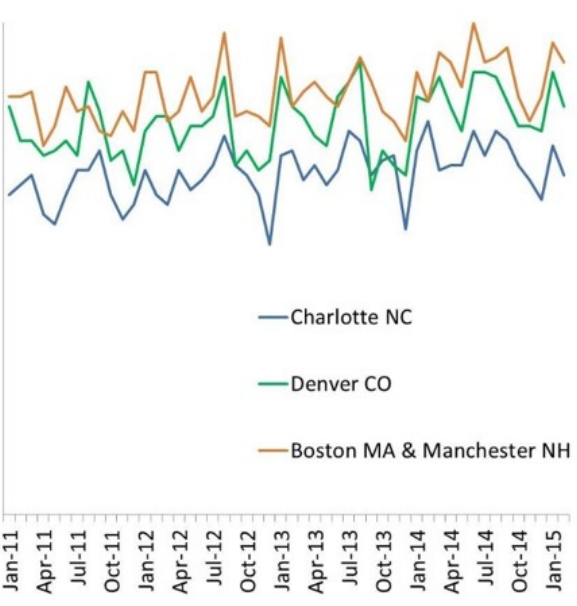

(b) three large town

(populations over 500,000)

Figure 2: Trends of public attention to dental care represented by Google search queries for the word "dentist". Google search queries for (a) three relatively small and (b) three relatively large US cities. The maximum value of queries is represented by 100 and other values are respective percentages of the maximum. Note: The public attention patterns to the word "dentist" show oscillatory behaviors. The amplitude of oscillation is larger in smaller towns.

small and large cities suggests that these patterns can be originated from a "Word Of Mouth" process in which people attract each other's attention to oral health. The different patterns in the small and large cities can be regarded to the different social network properties. In small towns, the distance between people in the social network is smaller than those in large cities which helps a faster "Word Of Mouth" process in the society. Regarding the search for the "dentist" as a sign of public attention to dental visits, it also suggests that these oscillations may have the same source as the cyclic behaviors in the number of dental visits (like Figure 1 .

1.7 Considering the importance of understanding collective dental care behaviors as a social phenomenon, we raise a question: can simple decision making rules at individual level result in oscillatory demand for routine visits? In order to answer this question, from a methodological perspective, by taking a dynamic modeling approach, we develop an agent-based model that aims to model individuals' routine dental check-ups behavior. Our approach offers another example of how dynamic modeling can be used to study complex patterns especially in public health arena (Auchincloss \& Roux 2008; Brookmeyer et al.2014; Day et al. 2013; Gorman et al. 2006. Laskowski et al. 2011; Leischow \& Milstein 2006). The model proposes a "Word Of Mouth" process in which people promote each other to go for dental visit through social networks.

1.8 Based on a simulation model, this paper postulates that: 1) given simple rules about how individuals (agents) go to dentists and also interact through social networks, it is likely to observe oscillatory patterns in the societal level. 2) Such a cyclic pattern is a function of social network characteristics that connects patients to each other. Our theory does not aim at explaining all reasons for routine dental visits (or any other routine medical visits), and does not aim at rejecting any previously stated reason for how individuals seek healthcare. However, it provides a novel "endogenous" theory of demand for routine dental visits, a theory that focuses on social dynamics of a preventive healthy behavior. 3) Finally, focusing on the fraction of people involved in web based activities, we compare the model results to an empirical data from Google Trends. This comparison demonstrates the oscillatory pattern of public attentions to oral and dental health issues, as well as the importance of routine visits that, can be predicted by the proposed model. In addition it indicates that the model is able to successfully explain the population dependency of these patterns for different US towns (Figure 2 .

\section{Modeling}

2.1 In order to reproduce the observed oscillatory pattern of dental visit demand, here an agent-based model is proposed. As previously mentioned in the introduction section, the cyclic pattern has specific properties: it is population dependent (Figure 2) and the period of the oscillations is about a few months. To reproduce these properties, the model is built based on a "Word Of Mouth" process, where agents interact through specific social 


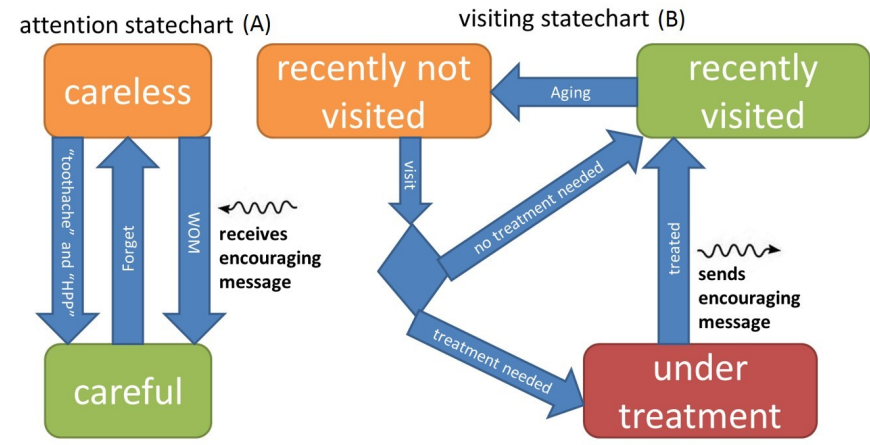

Figure 3: Statecharts of the agent-based model. The statechart on the left $(A)$ indicates the level of patient's attention to the necessity of routine visit and the statechart on the right (B) shows the visiting state. HPP stands for health promotion programs, and WOM stands for word of mouth.

networks. In addition, to simulate the cyclic behavior with a period of few months, we should also consider a delay of almost the same time period in which the simulation almost returns to its initial condition. Fortunately, there is a definite dental clinical delay for this time period which is related to necessary time intervals between routine dental visits advised by dentists. The visiting interval of about six months is assumed to be the effective interval for which the dental health status might become at risk (Frame et al. 2000).

2.2 The basic structure of the proposed model is as follows: In each time period, some people (agents) go to dentist. The decision to visit a dentist is influenced by individuals' state of attention to dental health (being careless or careful about their dental health), and whether or not they have recently (within the last six months) visited a dentist. When someone visits a dentist in order to do a check-up, it is possible that, he/she goes under treatment. It is assumed that people who have recently been under treatment encourage others to visit a dentist.

2.3 We run the model for different population sizes, network structures, and contact ratios as described in the model parameters section.

2.4 For coding and simulation purposes, AnyLogic software is used. In the following, we describe detailed formulation and coding of decision rules and network structures of the model. The model code can be found at: https://www .openabm.org/model/4927/version/1/view

\section{Decision rules}

2.5 Figure 3 depicts a flowchart (statechart) of an agent's decision making model. In each time period, two statecharts will indicate each agent's status. First is the state of attention about dental care (statechart A) which includes two conditions: careful (CF) and careless (CL). Second statechart indicates the agents visiting state (statechart B) where in each time period, each patient is in one of the states of recently visited (RV), recently not visited (RNV) and under treatment (UT). Thus for any agent $i$ and time $t$ we have:

$$
\begin{gathered}
A_{i, t} \in\{C F, C L\} \\
B_{i, t} \in\{R V, R N V, U T\}
\end{gathered}
$$

where $A_{i, t}$ and $B_{i, t}$ represent states of agent i at time t regarding her level of attention to dental care and visiting state, respectively.

\section{Transitions within attention states (statechart A)}

2.6 People's attention to dental care (statechart A) can change between $C L$ and $C F$. In our model, state of attention can increase (change from $C L$ to $C F$ ) through toothache, public Health Promotion Programs $(H P P)$, and word of mouth $(W O M)$. The probability of transition due to toothache or $H P P$ is represented by $\beta$. The WOM transition happens whenever the agent receives an encouraging message from her friend. Therefore, we can write:

$$
P\left(A_{i, t}=C F \mid A_{i, t-1}=C L\right)=\max \left(W O M_{i, t}, \beta\right)
$$


where $W O M_{i, t}=1$ if agent $i$ has received an encouraging message to seek dental care at time $t$, and is zero otherwise.

$$
W O M_{i, t}= \begin{cases}1, & \text { if } \exists j \mid S_{j i, t-1}=1 \\ 0, & \text { Otherwise }\end{cases}
$$

where $S_{j i, t-1}=1$ if agent $j$ sends encouraging message to $i$ at time $t-1$.

2.7 The level of attention deteriorates (changes from $C F$ to $C L$ ) with a delay of time to forget, $\tau$, formulated as following:

$$
\tau=M \tau_{N}
$$

where $\tau_{N}$ is the normal time to forget and $M$ is a multiplier, which represents effects of intensity of recent treatment on reminding the necessity of dental health. The transition from $C L$ to $C F$ can be expressed in terms of the transition probability as follows:

$$
P\left(A_{i, t}=C L \mid A_{i, t-1}=C F\right)=\left\{\begin{array}{lll}
1, & \text { if } & T_{C F} \geq \tau \\
0, & \text { if } & T_{C F}<\tau
\end{array}\right.
$$

where $T_{C F}$ is the time since the agent has become careful in her last transition.

\section{Transitions within visit states (statechart B)}

2.8 As mentioned before, agents are categorized into three groups: recently not visited (RNV), recently visited (RV), and currently under treatment (UT). Agents in RNV state are the ones who have not visited dentist in the past six months. Agents in the $R V$ state visited dentist at least once in the past six months. Agents in the $U T$ stage are currently under treatment.

2.9 In the model agents' decision to visit a dentist $\left(V_{i, t}=1\right)$ follows a simple rule: An agent visits a dentist if her attention and visiting states are respectively careful $\left(A_{i, t-1}=C F\right)$ and recently not visited dentist $\left(B_{i, t-1}=\right.$ $R N V)$. Mathematically:

$$
V_{i, t}= \begin{cases}1, & \text { if } \quad A_{i, t-1}=C F \quad \text { and } \quad B_{i, t-1}=R N V \\ 0, & \text { otherwise }\end{cases}
$$

2.10 The transition probabilities can be written as:

$$
\begin{aligned}
& P\left(B_{i, t}=R N V \mid B_{i, t-1}=R V\right)= \begin{cases}1, & \text { if } T_{R V} \geq 6 \text { months } \\
0, & \text { otherwise }\end{cases} \\
& P\left(B_{i, t}=U T \mid B_{i, t-1}=R N V\right)= \begin{cases}R T, & \text { if } V_{i, t}=1 \\
0, & \text { otherwise }\end{cases} \\
& P\left(B_{i, t}=R T \mid B_{i, t-1}=R N V\right)=\left\{\begin{array}{ll}
1, & \text { if } V_{i, t}=1 \\
0, & \text { otherwise }
\end{array} \text { and } B_{i, t} \neq U T\right. \\
& P\left(B_{i, t}=R T \mid B_{i, t-1}=U T\right)= \begin{cases}1, & \text { if } T_{U T} \geq T_{t} \\
0, & \text { otherwise }\end{cases}
\end{aligned}
$$

where $T_{R V}$ and $T_{U T}$ represent the time from when the agent's states has changed to $R V$ and $U T$ respectively, in her last transition. Whenever an agent visits a dentist, with some probability called required treatment $(R T)$, she will need treatment. Treatment time $\left(T_{t}\right)$, indicates how much time is needed to cure the agent. In addition, we have considered whenever an agent changes its state from $U T$ to $R V$, she will send an encouraging message to some randomly chosen friends of her.

$$
S_{j i, t}= \begin{cases}1, & \text { if } i \in R C_{m, j, t} \text { and } B_{i, t}=R V \text { and } B_{i, t-1}=U T \\ 0, & \text { otherwise }\end{cases}
$$

in which $R C_{m, j, t}$ is a set of $m$ randomly selected agents from the neighbors of agent $j$ in the social network. In other words, $m$ is the number of agent $j$ 's friends that she will encourage them after her treatment. We have varied $m$ in our simulations to investigate the effect of word of mouth in the collective behavior. 
2.11 We should emphasize that, focusing on the demand side, we have ignored the supply restrictions in our model. It should be mentioned that considering the limited supply side would probably smoothen the oscillations.

\section{Social network structures}

2.12 We used three major social network structures of Random, Ring Lattice, and Small World in our simulations, as described in the following.

\section{Random network}

2.13 In this structure each node (agent) is assumed to be randomly connected to a certain number of other nodes. Nodes are connected to any other one with the same probability (Figure 4). In our simulations all nodes are assumed to be randomly connected to $L=10$ other nodes on average. This means that any agent is connected to 10 other agents as friends (on average) and encourages $m$ number of them $(m \leq 10)$ to visit a dentist. We used AnyLogic pre-defined Random network standard structure to create this network. AnyLogic uses Erdős and Rényi (ER) random graph model to create the Random structure. In this model, distribution of each node's degree is binomial. In our case, since we have large number of nodes and also the mean degree of these nodes was constant, one can show the binomial distribution to be approximately equivalent to Poison distribution Newman 2010). In addition, in order to analyze the sensitivity of the model to the homogeneity of networks, we added (or subtracted) each node's degree by a normal variable with an average equals to 0 and variance equals to 5 . This addition (or subtraction) was performed by adding (or removing) a link between the node and another random node.

\section{Ring Lattice network}

2.14 In this structure, nodes are placed on a ring and each node is connected to a definite number of its neighbors. Connections in this type of network are local and there is no long range connection within the network structure (Figure 4). In these simulations, all nodes are assumed to be connected to 10 neighbors and they send encouraging messages to $m$ neighbors. We used AnyLogic pre-defined Ring Lattice network standard structure to create the network. The algorithm for creating a Ring Lattice network with nodes degree equal to 10 is as follows: first, a ring layout of agents is built, then we start from the first agent on the ring and connect it to its next 5 neighbors (clockwise). Repeating the process for all agents, each one will be finally connected to 10 adjacent agents, equally divided on its both sides on the ring. In order to analyze the sensitivity of the model to the homogeneity of the network structure, we then modify the network by adding (or removing) normal random number, with average 0 and variance 5, of edges to (or from) each node. In order to maintain the general structure of Ring Lattice network, when a link is removed, we choose the last connection in clockwise direction of that particular agent and when adding a link, we connect that agent to its last clockwise not connected neighbor.

\section{Small World network}

2.15 In this structure, connections are mostly local while it permits a certain fraction of connections to be long ranged. To be more specific, this network is based upon Ring Lattice network where each node is connected to a certain number of its neighbors. Then a given percent of local connections are replaced by connections between randomly chosen nodes with no limitation on their distance. Therefore, a Small World network structure can be considered as a combination of Ring Lattice and Random network (Figure 4]. We used the pre-defined Small World network in AnyLogic assuming 10 connections for each agent and 5 percent of the connections to be random $(\kappa=0.05)$. For creating this structure, AnyLogic follows Watts-Strogatz mechanism Watts \& Strogatz 1998). According to this mechanism, first Ring Lattice network should be created with a constant degree of each node as described before. Then for each node $\mathrm{i}$ an edge $\left(n_{i}, n_{j}\right)(i<j)$ is selected with probability $\kappa$ and it is rewired with $\left(n_{i}, n_{k}\right)$ where $\kappa$ is chosen uniformly at random. In order to analyze the sensitivity of the model to the homogeneity of the network structure, we then added (or removed) a normal random number, with an average of 0 and a variance of 5 , of edges to (or from) each node. 

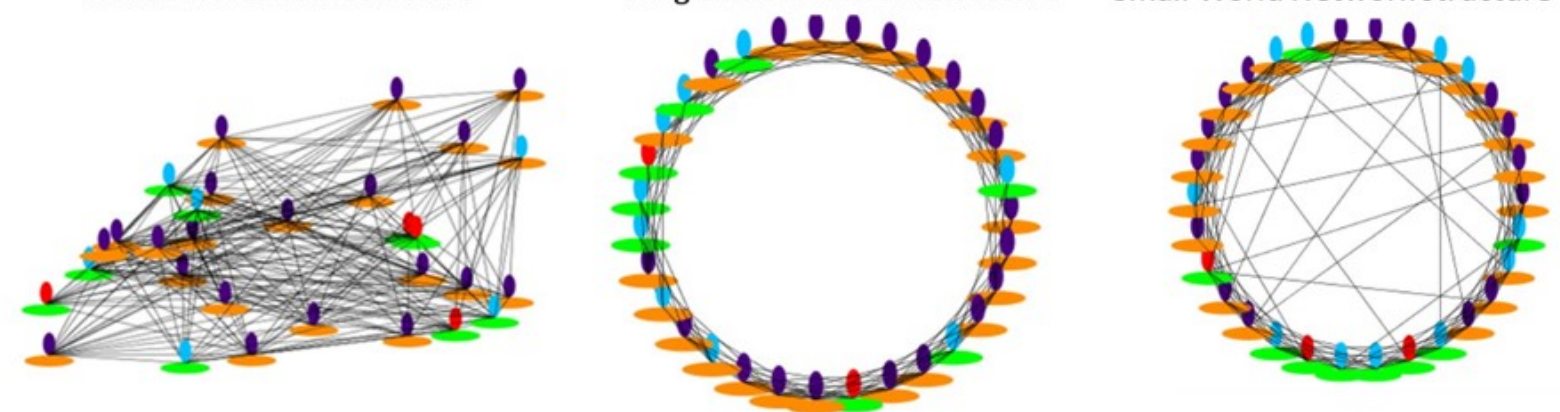

Figure 4: Schematic of different types of network structures. Agents with orange and green body (the lower oval) are careless and careful respectively. Agents with purple, blue and red head (the upper oval) are $R N V$, $R V$ and $U T$, respectively.

\begin{tabular}{lll}
\hline Parameter & Description & Value \\
\hline Visit Time $\left(T_{0}\right)$ & Proper period between successive routine visits. & 6 months \\
\hline Toothache \& HPP Rate $(\beta)$ & $\begin{array}{l}\text { Probability of a careless person become } \\
\text { careful by toothache or public advertisement. }\end{array}$ & 0.025 \\
\hline Contacts After Treatment $(m)$ & $\begin{array}{l}\text { Number of people who are encouraged } \\
\text { to visit a dentist by a person who } \\
\text { has recently been treated. }\end{array}$ & Varied between 0 to 10 \\
\hline Forget Time $\left(\tau_{N}\right)$ & $\begin{array}{l}\text { Normal time for a careful person to forget } \\
\text { and become careless. }\end{array}$ & $\begin{array}{l}\text { Normal random variable } \\
\text { with average of } 1 \text { month } \\
\text { and variance of } 0.5 \text { month }\end{array}$ \\
\hline Treatment Forget Multiplier $(M)$ & $\begin{array}{l}\text { Forget Time is multiplied by this value when the } \\
\text { person has been recently treated. }\end{array}$ & 3 \\
\hline Treatment Requirement Probability $(R T)$ & The probability of requiring a treatment during a visit. & 0.5 \\
\hline Treatment Time $\left(T_{t}\right)$ & Duration of treatment. & $\begin{array}{l}\text { Normal random variable } \\
\text { with average of } 0.5 \text { month } \\
\text { and variance of } 0.25 \text { month }\end{array}$ \\
\hline Total Population $(N)$ & Total population & Varied 100 - 20000 \\
\hline Connection Number $(L)$ & Average number of connections of each individual & 10 people \\
\hline Small World Network Parameter $(\kappa)$ & $\begin{array}{l}\text { Fraction of non-local connections } \\
\text { in the Small World network. }\end{array}$ & 0.05
\end{tabular}

Table 1: Short description of the ABM model parameters and their values.

\section{Model parameters}

2.16 In total, we have simulated 115,000 experiments which comprise 3 network structures, 11 different values of contacts after treatment (0-10), 7 different populations (100, 200, 500, 1000, 2000, 5000 and 20000), and 500 different random seeds. All models were simulated for the time period of 500 months and simulation time step is one-tenth of a month ( 0.1 month). All other parameters and their values are presented in Table 1.

\section{Oscillation measure}

2.17 In order to assess the intensity of oscillations in the simulations, we focus on the number of careful people. This variable has a continuous behavior over time and other variables like the number of visits over time almost imitates the same pattern. In addition, as we will see in the following sections, this variable is used to fit the empirical data. 
2.18 The spectral density $P$ of the trends can be used as a measure to examine how much the patterns of the results are oscillatory (Bloomfield 2004), as in the following:

$$
P(f)=|F T\{x(t)\}|^{2}
$$

where $P$ is the spectral density, $f$ is the frequency and $F T x(t)$ is the Fourier Transform of the continuous time series $x(t)$ (here the trends of number of careful people).

2.19 In order to have a better measure for the magnitude of oscillations, in a specific frequency interval, we use the normalized spectral density integral $(N I)$ defined as follows:

$$
\begin{aligned}
I & =\int_{f_{1}}^{f_{2}}\left(P(f)-P_{0}(f)\right) \mathrm{d} f \\
I_{0} & =\int_{0}^{f_{\max }} P(f) \mathrm{d} f \\
N I & =\frac{I}{I_{0}}
\end{aligned}
$$

where $I$ is the spectral density integral between $f_{1}$ and $f_{2}$ (the frequency interval of interest), $I_{0}$ is the total spectral density integral and $N I$ is the normalized spectral density integral. $P_{0}(f)$ indicates the condition in which no agent sends encouraging message $(m=0)$. This small value is subtracted from $P(f)$ so that we just integrate, in the interval $f_{1}$ and $f_{2}$, over the spectral density relevant to $W O M$ and we exclude the effect of the random process due to toothache and $H P P$.

2.20 Considering the lower and upper bound for the frequency interval of interest to be $f_{1}=0.1$ ( $1 /$ Month) and $f_{2}=0.2$ (1/Month) we can calculate $N I$. This frequency range is relevant to oscillations period times from 5 to 10 months which contains the 6 months regular dental visiting intervals.

\section{Results}

\section{Random network}

3.1 The simulation results for population 500 and $\mathrm{m}$ equals to 4 and 10 for Random structure are shown in Figure 5. This figure clearly shows an oscillatory pattern and the oscillation intensity grows as the number of contacts after treatment increases. The period of the oscillations is almost 7-8 months.
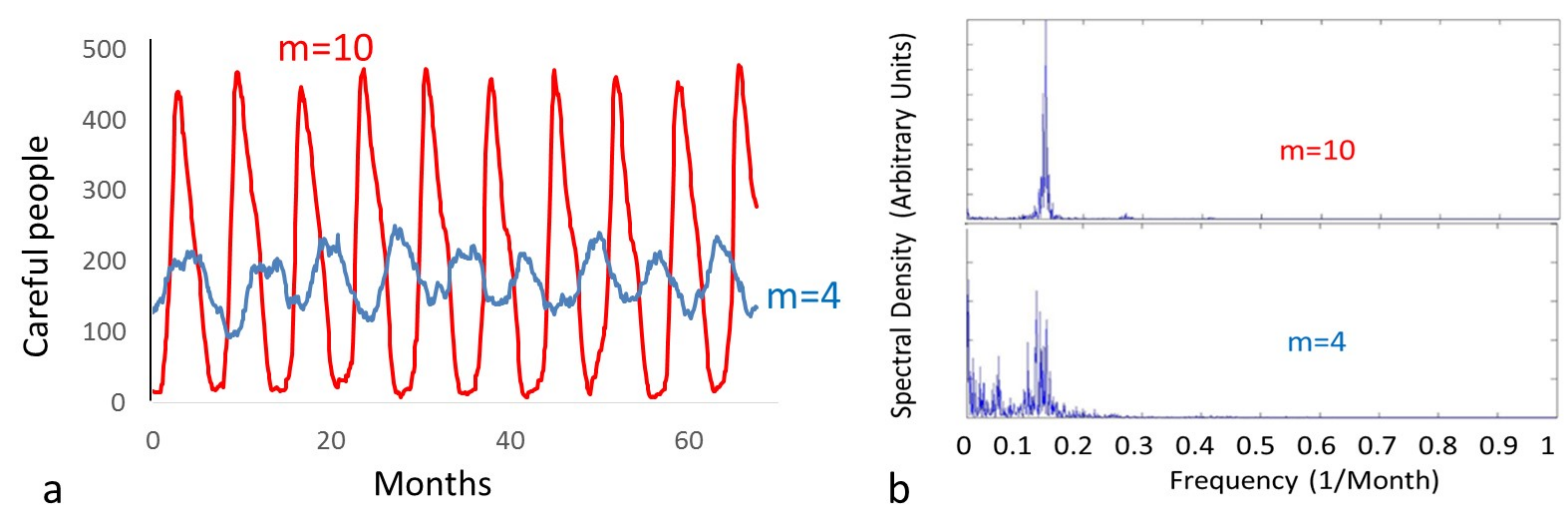

Figure 5: Time series and the relevant spectral densities of the trends of careful people in Random network simulations. Simulation results for a Random network with populations $m=4,10$ and population $N=500$. Note: a) Time series, (b) Spectral densities

3.2 The simulation results for the value of $\mathrm{N} /$ in the Random network for different populations are shown in Figure 6 a. For $m=0$, there is no collective oscillation and by increasing $m$, oscillations gradually appear especially 

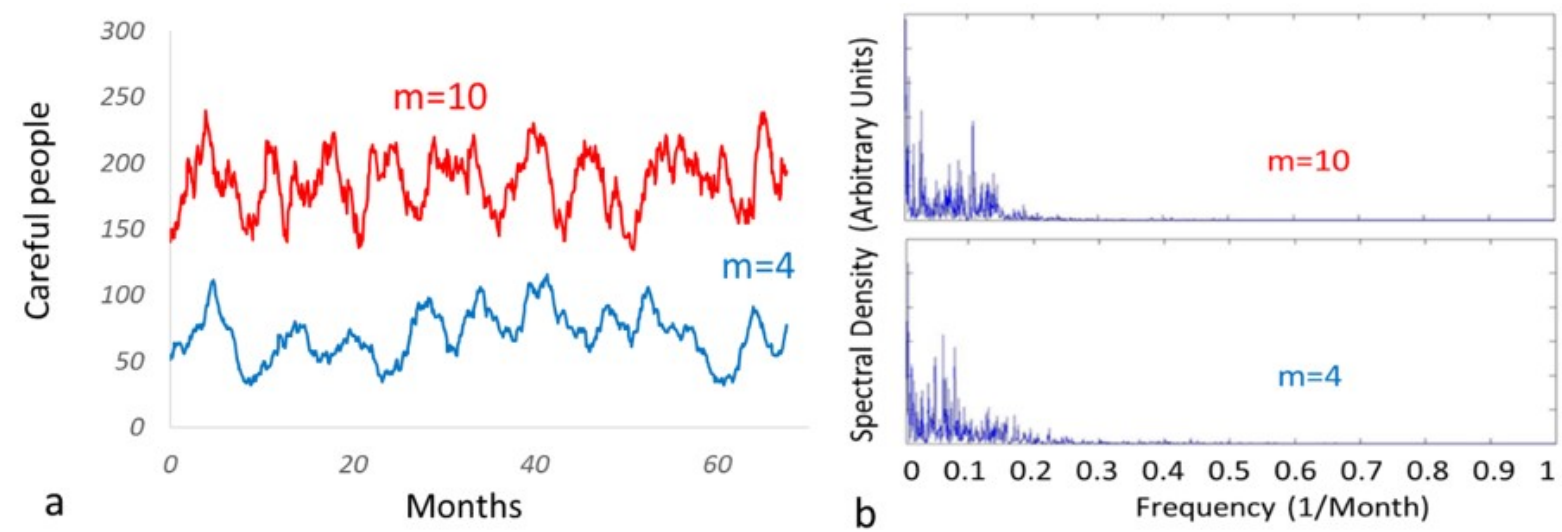

Figure 7: Time series and the relevant spectral densities of the trends of careful people in Ring Lattice network simulations. Simulation results for a Ring Lattice network with populations $\mathrm{m}=4,10$ and population $\mathrm{N}=500$. (a) Time series, (b) Spectral densities

for small populations. For different populations all graphs asymptotically tend to 0.4 . We will show in the next section that this is almost the maximum value of the normalized spectral density integral $N I_{\max } 0.4$.

3.3 Furthermore, the dependency of the oscillation intensity on the population is also illustrated in Figure 6 $\mathrm{p}$. This figure indicates that the simulation results are almost linear in a log-log diagram. We have also fitted the results for different values of "Contacts After Treatment" to a linear relation. These relations will be used in the following sections to compare the simulation results to empirical data. Interestingly, this figure illustrates that the results are independent of population size for larger values of $m$.

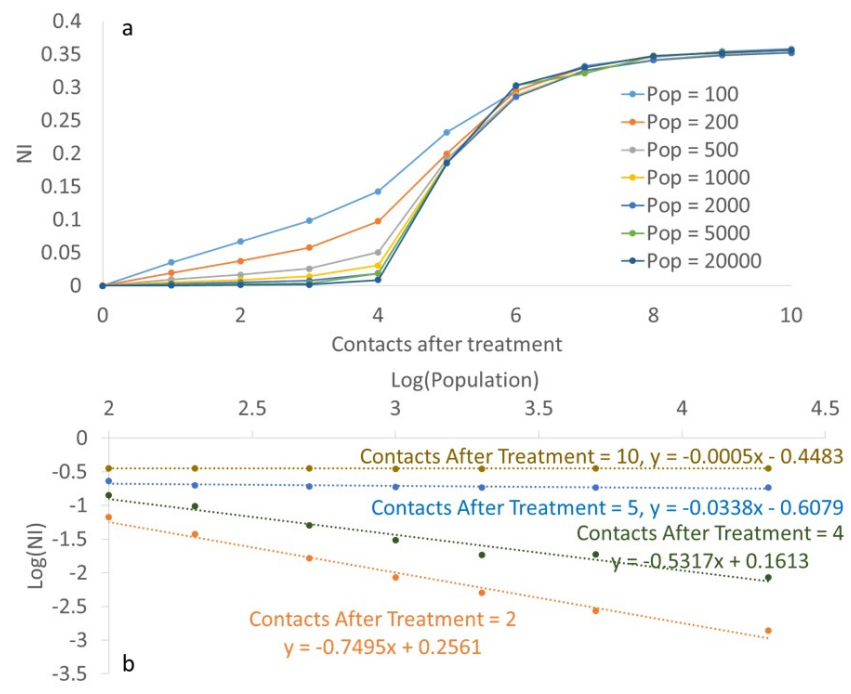

Figure 6: Simulation results for Random network structure. (a) Normalized spectral density for different "Contacts After Treatment", (b) log-log diagram showing the dependency of NI to population.

\section{Ring Lattice network}

3.4 The simulation results for $N I$ in Ring Lattice network is illustrated in Figure 8 a. According to this figure and by comparing it with Figure $6 \mathrm{~b}$, it can be seen that $N I$ values are much smaller than those for Random network. In addition, in contrast to Random network, the dependency of the oscillatory pattern on population, for larger values of $m$, is clearly observable (Figure $8 \mathrm{p}$ ). 


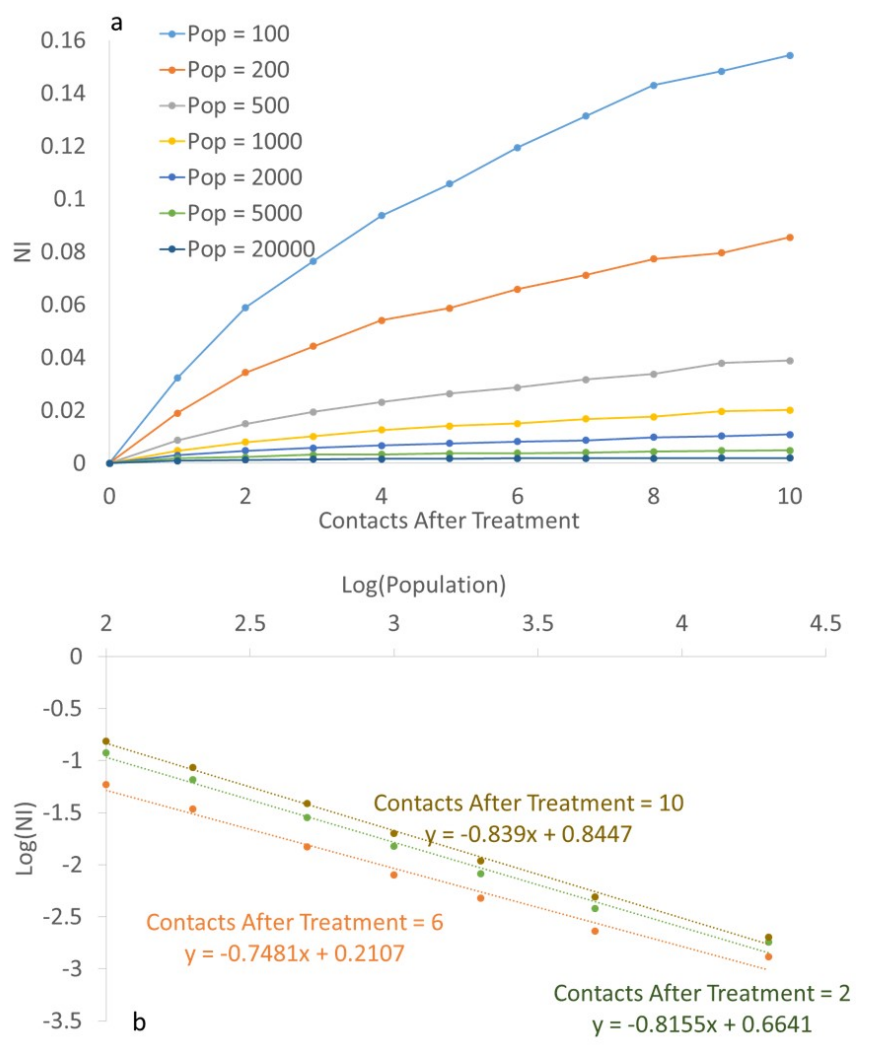

Figure 8: Simulation results for Ring Lattice. (a) Normalized spectral density for different "Contacts After Treatment", (b) log-log diagram showing the dependency of NI to population.

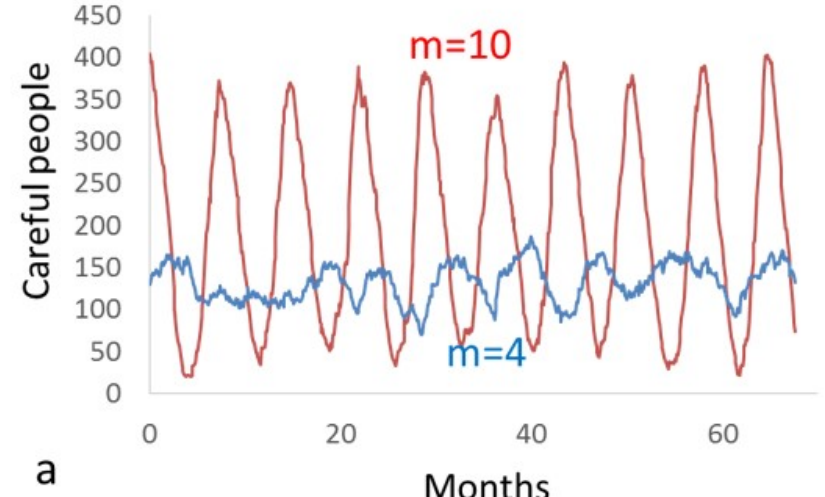

a

Months

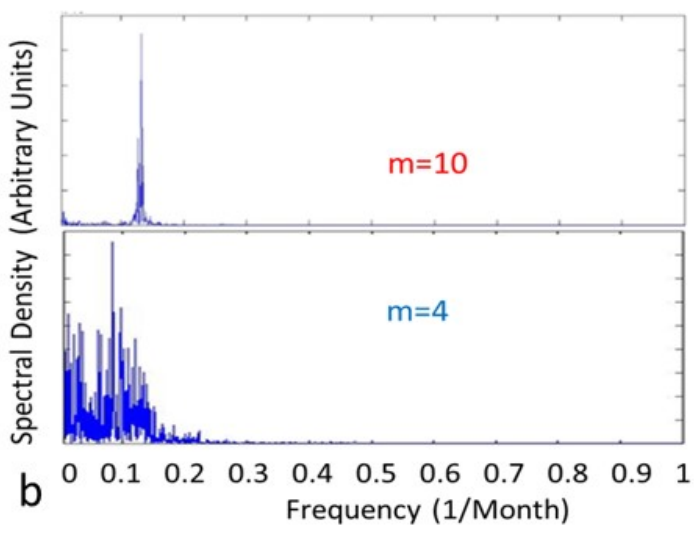

Figure 9: Time series and the relevant spectral densities of the trends of careful people in Small World simulations. Simulation results for a Small World network with populations $m=4,10$ and population $N=500$. (a) Time series, (b) Spectral densities

\section{Small world network}

3.5 The simulation results for population 500 and $\mathrm{m}$ equals to 4 and 10 for Small World structure is shown in Figure 9. In terms of pattern of connections, the Small World network is structurally between Random and Ring Lattice network structures. Therefore, it is expected that the speed of sending encouraging messages is in between those two extremes. This can be observed in the time series illustrated in Figure 9 when compared with two previous structures.

3.6 Simulation results for the Small World network (Figure 10a) show that for population values of few hundreds and for large values of $m, M l$ is large; while it has still a considerable difference to the maximum value 0.4 (as 


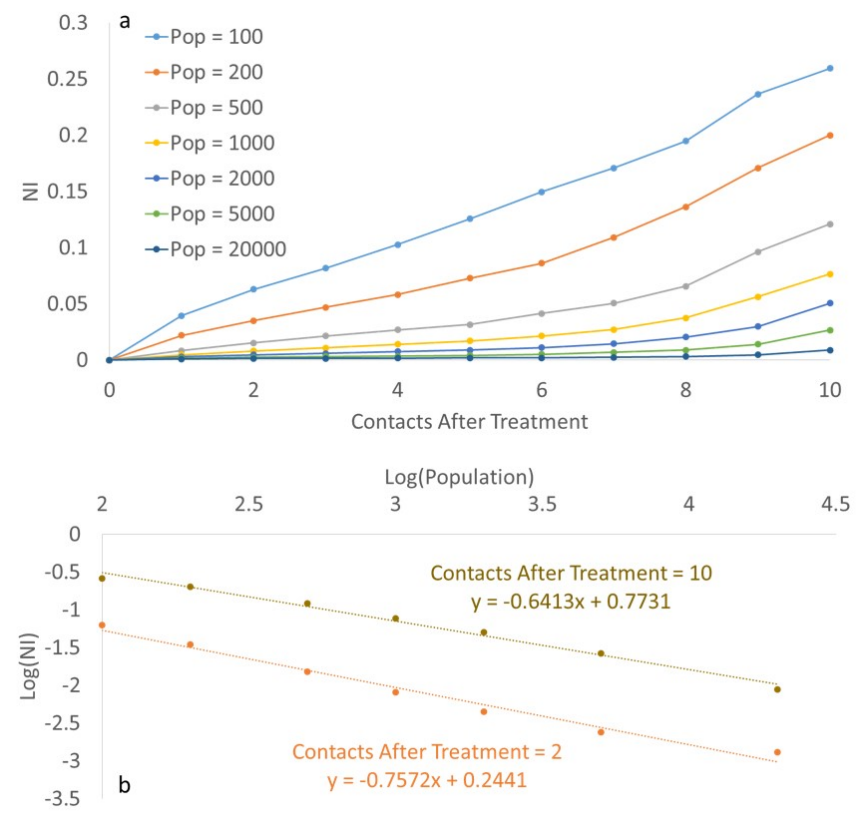

Figure 10: Simulation results for Small World. (a) Normalized spectral density for different "Contacts After Treatment”, (b) log-log diagram showing the dependency of $N I$ to population.
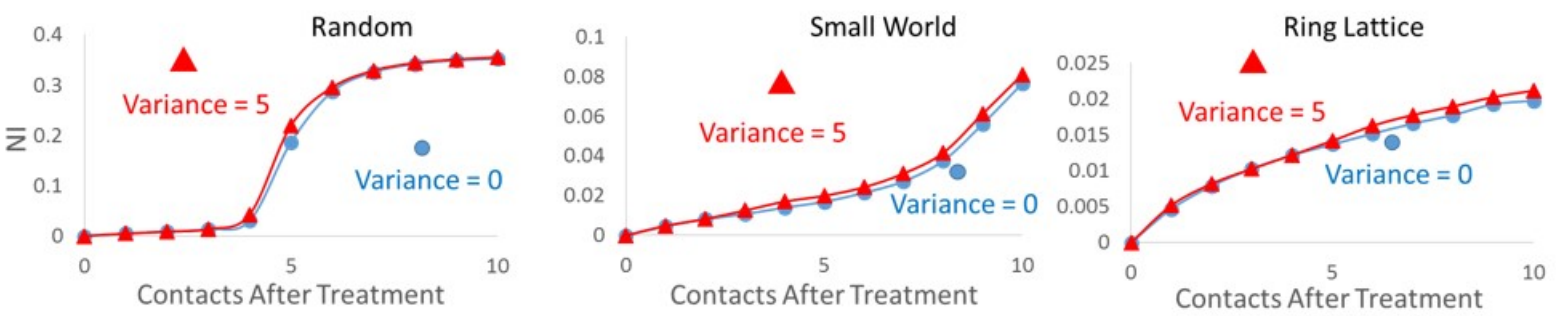

Figure 11: Sensitivity analysis of the results considering a normal random variable for the nodes' degree with variance 0 and 5 . The population of the network was set to 1000 .

calculated in the next section). Moreover, Figure $10 \mathrm{p}$ indicates that for small values of $m, \mathrm{MI}$ strongly depends on population and this dependency gradually decreases as $\mathrm{m}$ increases.

\section{Sensitivity to the network homogeneity}

3.7 In order to analyze the sensitivity of the results to the homogeneity of the nodes' degree, we assume each node's degree to be a normal variable with average 10 and variance 5. Figure 11 illustrates the comparison with the results of the homogenous model for different network structures.

3.8 As illustrated in Figure 11. the variation from the homogenous network is not significant, however this variation is relatively larger for the Ring Lattice network. The oscillations are a bit larger for the Ring Lattice network which can be due to the fact that the random distribution adds some longer links to the network.

\section{Summary results}

3.9 The above mentioned results are summarized in Table 2. This table presents qualitative results on collective behavior for different values of population and "Contacts After Treatment" $(m)$. 


\begin{tabular}{|c|c|c|}
\hline & Low $m$ & High $m$ \\
\hline Low population & $\begin{array}{l}\text { Oscillation is observed in all network structures. } \\
\text { The magnitude depends on population. }\end{array}$ & $\begin{array}{l}\text { Oscillation depends on the network structure. } \\
\text { (a) Random network: maximum oscillation } \\
\text { (b) Ring Lattice: small oscillation } \\
\text { (c) Small World: large oscillation }\end{array}$ \\
\hline High population & $\begin{array}{l}\text { Oscillation depends on the network structure. } \\
\text { (a) Random network: small oscillation } \\
\text { (b) Ring Lattice: no oscillation } \\
\text { (c) Small World: no oscillation }\end{array}$ & $\begin{array}{l}\text { Oscillation depends on the network structure. } \\
\text { (a) Random network: maximum oscillation } \\
\text { (b) Ring Lattice: no oscillation } \\
\text { (c) Small World: large oscillation }\end{array}$ \\
\hline
\end{tabular}

Table 2: Summary of observed results in simulations.

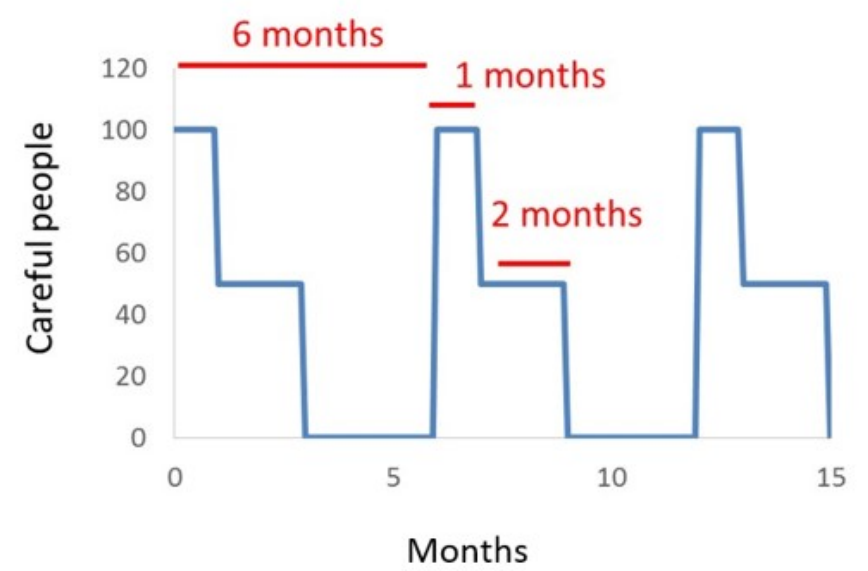

Figure 12: Time series for careful people in the extreme case when all people are promoted together. Note: We have assumed whole population contribute in the collective behavior and people become careful all together. Total population is set to 100 .

\section{Theory and Discussion}

\section{Oscillation origin}

4.1 The origin of the observed simulated oscillations can be explained as follows. When there are many careful people, some of them randomly have a visit due to toothache or public HPP. If they need and receive treatment, they will talk about their experience with some friends and therefore more people will have visit afterwards. On the other hand, since agents usually become careless after a while (forget), the same iteration will happen again.

4.2 In extreme cases in which all people contribute in the collective behavior and become aware immediately all together, time series profile for careful people would be as illustrated in Figure 12 In this case, all people in the population are promoted, for visiting, together and go for check-ups. After one month in average, half of them who were not under treatment become careless, while the other half become careless after three months. $N I_{\max }$ for this extreme case is then found as follows:

$$
N I_{\max } \sim 0.4
$$

This relation roughly gives an upper bound for $N I$ and as it was shown in results section, some of our simulation results converged to this value under specific conditions.

\section{Oscillation condition}

4.3 In order to find a rough approximation for the condition in which oscillation is observable, we consider the following scenario. Consider at time $=0$ all agents are careless and recently not visited a dentist. Then, randomly one of them becomes careful and excited enough to encourage friends (after treatment) and successively, in 


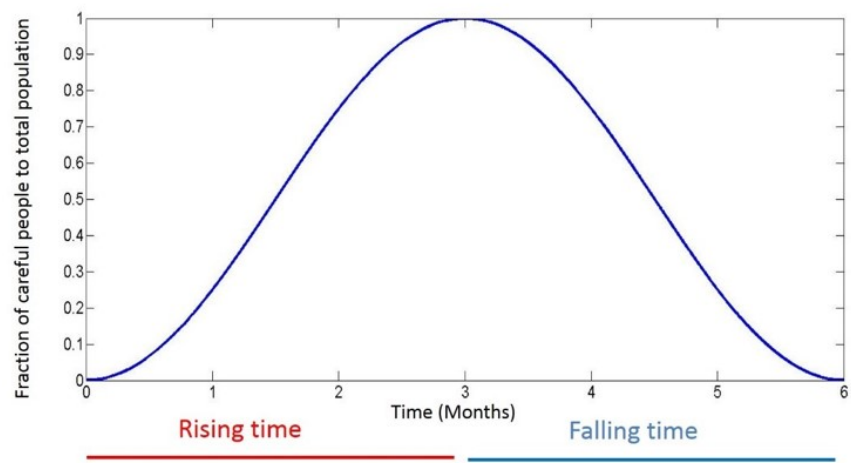

Figure 13: Schematic of the pattern of number of careful people when we have collective oscillatory behavior.Note: Most of the population are expected to become careful in the first three months. In the following sub sections, some simple relations for the oscillation condition in Random and Ring Lattice networks as two extreme situations are driven. Calculations for Small World networks are much more complicated, but we can simply consider it as a condition between Random and Ring Lattice networks.

chain reaction process of sending encouraging messages, a considerable amount of people become careful and go for check-ups. In order to observe the collective oscillatory pattern, with the $T_{0}=6$ months period, the chain reaction process speed must be large enough that in $\frac{T_{0}}{2}=3$ (the first half of the total $T_{0}=6$ months period) most of the population become careful. This is when the number of careful people is expected to rise in oscillatory pattern (Figure 13). Otherwise, if a considerable amount of people remain careless after the initial three months, then the rising in number of careful people will continue in the second half of $T_{0}$, at the time when the collective oscillation is expected to fall; the oscillatory pattern is destroyed.

\section{Random network}

4.4 To formulate the oscillation condition for Random network, we consider a stepwise process where each time step has a length of $T_{t}=$ "Treatment Time" assuming that at time $t=0$ all people were careless and recently not visited dentist. At that time, one randomly chosen person becomes careful, pays a visit to a dentist, and then goes under treatment. After time step $T_{t}$, this patient encourages her friends to go for visit and the same process, as the last step, starts with larger number of patients. Consider we have $n_{k}$ people who have already become careful and gone for visit until step $k$. From this population, $n_{k} \breve{n} n_{k-1}$ individuals became careful in the step $k$ and half of them (who need treatment) will encourage their friends in step $k+1$. Therefore, $n k+1$ can be calculated as follows:

$$
n_{k+1}=n_{k}+\frac{C}{2}\left(n_{k}-n_{k-1}\right)\left(1-\frac{n_{k}}{N}\right)+r\left(N-n_{k}\right) T_{t}
$$

where $C$ is the number of encouraging messages that an excited person sends to her friends and $r$ is the rate of "Toothache \& HPP" transition.

4.5 The second term on the right hand side of the equation, which indicates the new careful agents due to $W O M$, counts the number of encouraging messages, $\frac{C\left(n_{k}\right)-n_{k-1}}{2}$, multiplied by the probability that the message receiving agent has not received any message in the previous steps, $\frac{1-n_{k}}{N}$. The factor $\frac{1}{2}$ in the above relation arises since we have assumed that half of the agents (Required Treatment probability, $R T=0.5$ ) need treatment, and so will send encouraging messages. In addition, if there are $m$ "Contacts After Treatment" and $L$ network connections per agent (in our simulations $L=10$ ), the agent will send a message back to the person who has just recently encouraged her with probability $\frac{m}{L}$. So $C$ should be considered as $C=m-\frac{m}{L}$. The third term, in the above relation, counts the number of new careful agents due to random "Toothache \& HPP" transition during one step. In our simulations, the rate of "Toothache \& HPP" transition, $r$, was considered to be 0.025 .

4.6 To find a simple, at the same time rough, approximation one can assume the early fast growing stages of the chain reaction process, $\frac{n_{k}}{N}<<1$ and also assume $C>>1$ that means an agent sends encouraging messages to most friends. In this situation with $n_{k}>>n_{k-1}$ the discrete relation can be written as follow:

$$
n_{k+1}-n_{k}=\frac{C}{2} n_{k}+r N T_{t}
$$


4.7 Given that the total time for growing the number of careful people is about three months. this is much larger than the time length of each step ( $T_{t}=0.5$ months). Therefore, we can rewrite the above discrete relation in a continuous form as follows:

$$
\frac{d n}{d t}=\frac{C}{2 T_{t}} n+r N
$$

4.8 By solving this differential equation, we easily find the condition in which most of the population is covered (become careful) in the first half of the total $T_{0}=6$ months of the oscillation period.

$$
\ln \frac{C}{2 T_{t} r} \leq \frac{C T_{0}}{4 T_{t}}
$$

4.9 Interestingly this condition is independent of $N$. In other words, in Random networks the oscillatory collective behavior will occur in the society if the above non-equality is satisfied no matter how large the population is. The above inequality is satisfied for a critical $C$ value of $C_{\text {min }}=1.33$ which almost corresponds to a critical $m$ value of $m_{\min } \sim 1.5$. It means that if each agent encourages at least 1.5 friends the collective oscillation is observable.

4.10 These theoretical results can be compared with simulation results presented in Figure 6 . This Figures shows that for larger values of "Contacts After Treatment" $(m)$ oscillation intensity is independent of population which is in complete agreement with the theory. It also shows that for these large values of $m$, the value of $N I$ almost reaches its maximum value, $N I_{\max }=0.4$. The similarity between the oscillation's peaks shapes of Figure $5 \mathrm{p}$ (for $m=10$ ) and Figure 12 is observable.

4.11 Also it can be seen that the oscillation intensity becomes considerable for values of $m$ greater than 3. This shows that the theory underestimates the critical min value for the oscillation condition. This deviation can be due to the fact that in the approximations we have assumed that $C>>1$.

\section{Ring Lattice network}

4.12 For Ring Lattice network, agents are placed on a ring and individuals become careful due to WOM locally. In this structure, there are some careful regions on the ring that grow by their two end sides. Assuming that half of the individuals who receive the encouraging message will need treatment and send the subsequent messages, we simply assume that a local careful region grows with $\frac{C}{2}$ agents at each step. It should be noted that this assumption is just a rough approximation for which we have avoided sophisticated calculations. The differential equation for $n$ follows as:

$$
\begin{aligned}
\frac{d n}{d t} & =\frac{C}{2 T_{t}} D \\
\frac{d D}{d t} & =\frac{r N}{2}
\end{aligned}
$$

4.13 Where $D$ is the number of careful regions. The first equation calculates the growth of $D$ careful regions and the second equation is the rate in $D$ increment due to the random "toothache and HPP" transition. The $1 / 2$ factor in the second equation comes from the fact that half of the randomly becoming careful individuals do not need treatment and so will not create an active careful region. It should be stated that as $C$ is assumed to be large, and if the first randomly created active point of the arc is active, then the arc will remain active. It is also assumed that $n<<N$. For the condition in which most of the agents become careful in first $\frac{T_{0}}{2}$ we find:

$$
N \leq \frac{C}{4 T_{t}} T_{0}+\frac{N C r}{8 T_{t}}\left(\frac{T_{0}}{2}\right)^{2}
$$

4.14 The first term on the right hand side is relevant to the first careful region that is assumed to exist at $t=0$. The second term appears due to the consequent careful regions forming randomly due to toothache and $H P P$. In contrast to Random network, this inequality strongly depends on population. For the maximum value of $C=9$ we find a condition of $N<30$ satisfies the oscillation condition. These results are in consistent with simulation results (Figure 8 where the oscillation intensities are much smaller than Random network and strongly depends on population. The simulation results show that for $N=100$ we still have considerable oscillations. This deviation from theory can be due to the fact that the careful regions actually grow faster than the simple approximation rate $\frac{C}{2}$. It should also be noticed that for Small World network, as expected, Figure 10 shows 


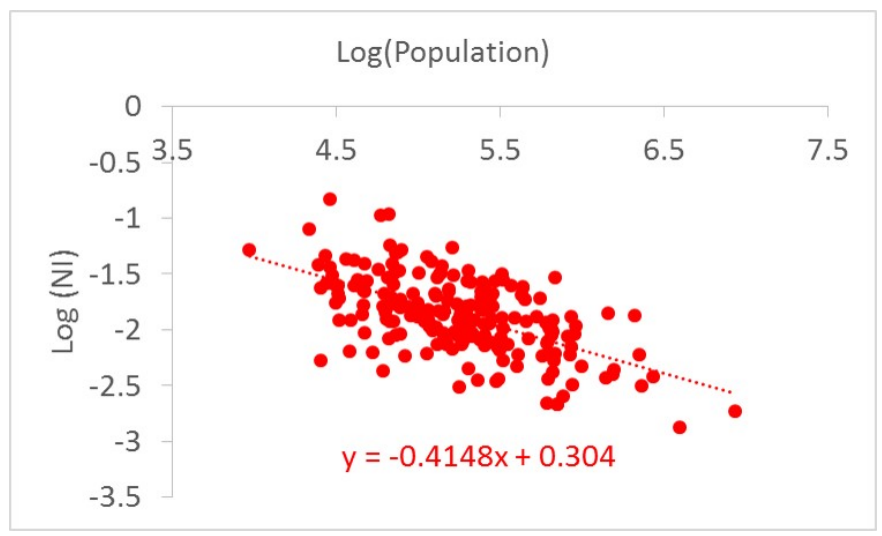

Figure 14: Data from Google Trends fitted to a linear relation between NI and Population in a log-log diagram.

that for small values of $C$ the oscillation intensity is population dependent while this dependency gradually reduces as $C$ increases. This result clearly reflects on the nature of the Small World network as a case between two extreme cases of Random and Ring Lattice networks.

\section{Comparison with Empirical Data}

5.1 To validate the model, the results are compared with an empirical data gathered from Google Trends service which reports the relative number of queries to search specific words by certain user defined time periods and locations. This service can be used to report the relative number of queries related to a specific category. In order to find a proper measure for the people's attention to oral health issues, choosing US society as a developed country with high level of access to Internet, we gathered the reports of the relative number of searches in the "Oral \& Dental Care" category for 208 cities in US since early 2004. The names of cities in addition to their population can be found in Appendix For each city, the report consisted of a time series showing the relative number of queries over time. The data indicates that oscillatory behavior and oscillations' intensity is population dependent. The relative reported numbers, showing the amount of searchers' attention to dental and oral health care issues, somehow reflect a measure of carefulness among the society. We assume that the relative amount of search queries is proportional to the number of careful people in our model. Calculating the power spectrum of the time series, we found the N/ values for each city and by knowing its population size we could compare the data to the model results. In order to find a relation between $\mathrm{N}$ and the population of the city, we found that the data best fits a linear relation in a log-log diagram. Figure 14 shows the fitted data and the linear regression statistical analysis results are illustrated in Table 3 . The slope of the linear equation is $-0.41 \pm 0.08$. Comparing the linear equations of the relevant model graphs (Figure $6 \mathrm{~b}$, Figure $8 \mathrm{~b}$, Figure $10 \mathrm{p}$ ), it turns out that the simulation results for Ring Lattice and Small World network decrease much faster, as the population increases, compared with Google Trends data. The slope of the linear equation for Ring Lattice network is almost -0.8 for all of the values of "Contacts After Treatment" while for Small World the slope is at most -0.64 , relevant to 10 contacts after treatment.

5.2 In the case of Random network, the simulations graphs cover a wide range of slope values from -0.8 to 0 . The slope $=-0.41$, relevant to the Google data, occurs for a value between 4 and 5 number of contacts after treatment. It shows that the Google Trends data can fit the Random network model for "Contacts After Treatment" of about 4-5. The Google Trends data is compared to the Random model in Figure 15. It should be mentioned that, in order to fit better to the data, we have shifted the empirical point for about 0.7 to the left on the log(population) axis. This means that the model well explains the data if we assume that $20 \%$ of the population actually involve in the network. This fraction value can be regarded as an estimation for the fraction of the society engaged in the Internet based activities; they are deeply influenced by Internet social networks and use Internet as a significant source of information.

\section{Conclusion}

6.1 There are some empirical facts that show the effect of peer connections on individuals' health behaviors. In 


\begin{tabular}{lcccccccc}
\hline & Coefficients & Standard Error & t Stat & P-value & Lower 95\% & Upper 95\% & Upper 95.0\% & Lower 95.0\% \\
\hline Intercept & 0.303961 & 0.20830 & 1.4592 & 0.14603 & -0.10673 & 0.71464 & -0.10673 & 0.71464 \\
X Variable & -0.4148 & 0.03950 & -10.5009 & $6.2 \mathrm{E}-21$ & -0.49268 & -0.33692 & -0.49268 & -0.33692 \\
\hline
\end{tabular}

Table 3: Regression analysis of the Google Trends Data.

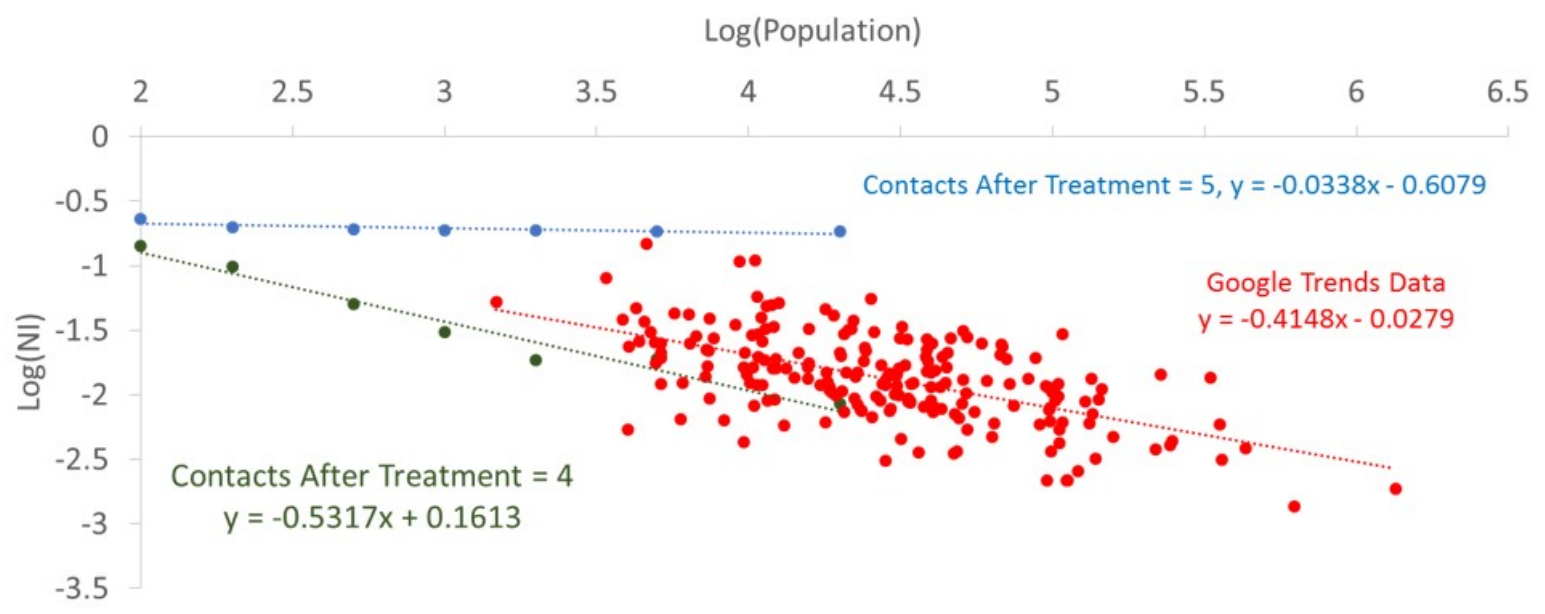

Figure 15: Simulation results fitted to linear relation between NI and population, in log-log diagram.

some cases, these individuals' behavior changes produce interesting collective behaviors, as emergent properties of the system, in the society level. One of these social emergences can be observed in the oscillatory pattern of the amount of demand for dental visiting in social networks. In the current work, an agent-based model is introduced to investigate this collective behavior. The model illustrates that this oscillatory pattern is strongly dependent upon the network structure and the number of effective connections. In Random networks, the oscillations appear as the number of effective contacts among agents exceeds a critical value of about 3 , and interestingly for larger values of effective contacts the oscillation intensity reaches its maximum no matter how large the population is. In Ring Lattice networks, where we have local connections, the oscillation intensity is much smaller than Random network. In addition, in contrast to Random network the oscillatory pattern is strongly dependent on population in the Ring Lattice network. For Small World network the results are somehow a combination of the Random and Ring Lattice networks. Comparing the model results to empirical data from Google Trends, it turns out that the results best fit to Random network model while we assume that $20 \%$ of people are involved in the network in each US city. This suggests that the model can be used focusing on the fraction of people involved in Internet social networks and activities. As an insight, the model predicts that the dental health service demand oscillations may grow as people are more influenced by Internet social networks which may lead to resource management problems. Of course it should be noted that the model results are based on restricted assumptions; the model has ignored many other participants like suppliers and also demands from other parts of the society whom not using internet which may become significant in specific situations.

\section{Acknowledgement}

This research was supported by Research Institute of Dental Sciences, Shahid Beheshti University of Medical Sciences. We must also thank Professor Navid Ghaffarzadegan from Virginia Tech University for his precious helps and advices during the research. 


\section{Appendix}

Table 4: 208 cities and regions in US for which the Google Trends data was gathered (Source: U.S. Census Bureau's 2014 Population Estimates).

\begin{tabular}{|c|c|}
\hline City & Population \\
\hline New York NY & 8491079 \\
\hline Los Angeles CA & 3928864 \\
\hline Chicago IL & 2722389 \\
\hline San Francisco, Oakland, San Jose CA & 2282029 \\
\hline Houston TX & 2239558 \\
\hline Dallas, Ft. Worth TX & 2093285 \\
\hline Philadelphia PA & 1560297 \\
\hline Phoenix AZ & 1537058 \\
\hline San Antonio TX & 1436697 \\
\hline San Diego CA & 1381069 \\
\hline Sacramento, Stockton, Modesto CA & 996874 \\
\hline Austin TX & 912791 \\
\hline Raleigh, Durham (Fayetteville) NC & 895737 \\
\hline Seattle, Tacoma WA & 873501 \\
\hline Jacksonville FL & 853382 \\
\hline Indianapolis IN & 848788 \\
\hline Columbus $\mathrm{OH}$ & 835957 \\
\hline Charlotte NC & 809958 \\
\hline Boston MA, Manchester NH & 766332 \\
\hline Minneapolis St. Paul MN & 704847 \\
\hline Washington DC (Hagerstown MD) & 699257 \\
\hline Detroit MI & 680250 \\
\hline El Paso TX & 679036 \\
\hline Tampa, St. Petersburg (Sarasota) FL & 666606 \\
\hline Denver CO & 663862 \\
\hline Cleveland, Akron (Canton) $\mathrm{OH}$ & 659677 \\
\hline Memphis TN & 656861 \\
\hline Fresno, Visalia CA & 645267 \\
\hline Nashville TN & 644014 \\
\hline Greensboro, High Point, Winston Salem NC & 630484 \\
\hline Albuquerque, Santa Fe NM & 627466 \\
\hline Baltimore MD & 622793 \\
\hline Oklahoma City OK & 620602 \\
\hline Portland OR & 619360 \\
\hline Las Vegas NV & 613599 \\
\hline Louisville KY & 612780 \\
\hline Miami Ft. Lauderdale FL & 606345 \\
\hline Milwaukee WI & 599462 \\
\hline Tucson (Sierra Vista) AZ & 571778 \\
\hline Colorado Springs, Pueblo CO & 554253 \\
\hline Norfolk, Portsmouth, Newport News VA & 524394 \\
\hline Kansas City MO & 470800 \\
\hline Atlanta GA & 456002 \\
\hline Omaha NE & 446599 \\
\hline Wichita, Hutchinson KS & 430055 \\
\hline Ft. Smith, Fayetteville, Springdale, Rogers AR & 429328 \\
\hline Harlingen, Weslaco, Brownsville, McAllen TX & 425157 \\
\hline Orlando, Daytona Beach, Melbourne FL & 403873 \\
\hline Tulsa OK & 399682 \\
\hline New Orleans LA & 384320 \\
\hline
\end{tabular}


Bakersfield CA 368759

Honolulu HI 350399

Lincoln \& Hastings, Kearney NE 330380

Cedar Rapids, Waterloo lowa City \& Dubuque IA 329410

Flint, Saginaw, Bay City MI 328163

Grand Rapids, Kalamazoo, Battle Creek MI 321547

Corpus Christi TX 320434

St. Louis MO $\quad 317419$

$\begin{array}{ll}\text { Lexington } \mathrm{KY} & 310797\end{array}$

Pittsburgh PA $\quad 305412$

Anchorage AK $\quad 301010$

Cincinnati OH 298165

Yakima, Pasco, Richland, Kennewick WA 292445

Huntsville, Decatur (Florence) AL 283882

Waco, Temple, Bryan TX 281872

Toledo OH 281031

Providence RI, New Bedford MA 273999

Des Moines, Ames IA 272486

Mobile AL, Pensacola (Ft. Walton Beach) FL 269301

Buffalo NY 258703

Ft. Wayne IN 258522

Hartford \& New Haven CT 254987

Laredo TX 252309

Champaign \& Springfield, Decatur IL 252086

Tyler, Longview(Lufkin \& Nacogdoches) TX 250899

Richmond, Petersburg VA 250554

Madison WI $\quad 245691$

Lubbock TX 243839

Little Rock. Pine Bluff AR 243038

Odessa. Midland TX 242634

Santa Barbara, Santa Maria, San Luis Obispo CA 241336

Reno NV 236995

Baton Rouge LA 228895

Montgomery (Selma) AL 220295

Boise ID $\quad 216282$

Greenville, Spartanburg SC, Asheville NC, Anderson SC 214840

Albany, Schenectady, Troy NY 214412

Birmingham AL $\quad 212247$

Spokane WA 212052

Rochester NY 209983

Tallahassee FL, Thomasville GA 206807

Wichita Falls TX \& Lawton OK 202131

Columbus GA 200887

Shreveport LA $\quad 198242$

Amarillo TX 197254

Augusta GA 196741

Peoria, Bloomington IL 194558

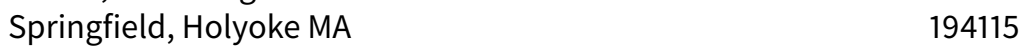

Salt Lake City UT 190884

Monterey, Salinas CA 184953

Knoxville TN 184281

Sioux Falls(Mitchell) SD 184279

Chico, Redding CA 180773

Roanoke, Lynchburg VA 178475

Harrisburg, Lancaster, Lebanon, York PA 177822

Chattanooga TN 173778

Beaumont, Port Arthur TX 172133

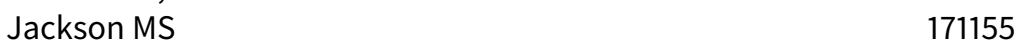


Springfield MO 165378

Minot, Bismarck, Dickinson(Williston) ND 163777

Eugene OR 160561

Columbia, Jefferson City MO 160038

Macon GA 153691

South Bend, Elkhart IN 152611

Alexandria LA 150575

Rockford IL 149123

West Palm Beach Ft. Pierce FL 147632

Savannah GA 144352

Syracuse NY 144263

Abilene, Sweetwater TX 141709

Dayton OH 141003

Davenport IA, Rock Island, Moline IL 139483

Yuma AZ, El Centro CA 137163

Tri, Cities 134352

Columbia SC 132067

Charleston SC 130113

Greenville, New Bern, Washington NC 129932

Gainesville FL $\quad 128460$

Topeka KS $\quad 127215$

Lafayette LA 126066

Fargo, Valley City ND 122539

Evansville IN 120346

La Crosse, Eau Claire WI $\quad 118122$

Biloxi, Gulfport MS 116734

Green Bay, Appleton WI $\quad 116147$

Wilkes Barre, Scranton PA 116095

Lansing MI 114620

Wilmington NC $\quad 113657$

Idaho Falls, Pocatello ID $\quad 112983$

Duluth MN, Superior WI 112943

Billings MT 108869

Medford, Klamath Falls OR 99676

Erie PA $\quad 99452$

Charleston, Huntington WV $\quad 99211$

San Angelo TX $\quad 98975$

Ft. Myers, Naples FL $\quad 91886$

Portland, Auburn ME $\quad 89578$

Bend OR $\quad 84080$

Sioux City IA $\quad 82511$

Grand Junction, Montrose CO 79255

Cheyenne WY, Scottsbluff NE $\quad 77720$

Paducah KY, Cape Girardeau MO, Harrisburg, Mount Vernon IL 77615

St. Joseph MO 76967

Butte, Bozeman MT $\quad 76340$

$\begin{array}{ll}\text { Albany GA } & 75769\end{array}$

Lake Charles LA $\quad 74889$

Rapid City SD $\quad 72638$

Jonesboro AR $\quad 72210$

Burlington VT, Plattsburgh NY $\quad 71552$

Casper, Riverton WY 71039

Columbus, Tupelo, West Point MS 70029

Missoula MT $\quad 69821$

Quincy IL, Hannibal MO, Keokuk IA $\quad 69390$

Monroe LA, El Dorado AR 67953

Florence, Myrtle Beach SC $\quad 67953$

$\begin{array}{ll}\text { Jackson TN } & 67319\end{array}$ 


\begin{tabular}{|c|c|}
\hline Dothan AL & 67180 \\
\hline Victoria TX & 66094 \\
\hline Johnstown, Altoona PA & 65742 \\
\hline Youngstown $\mathrm{OH}$ & 65062 \\
\hline Joplin MO, Pittsburg KS & 64698 \\
\hline Rochester MN, Mason City IA, Austin MN & 63576 \\
\hline Bowling Green KY & 62479 \\
\hline Utica NY & 61332 \\
\hline Terre Haute IN & 60956 \\
\hline Hattiesburg, Laurel MS & 60688 \\
\hline Great Falls MT & 59152 \\
\hline Sherman TX, Ada OK & 57073 \\
\hline Harrisonburg VA & 52478 \\
\hline Greenwood, Greenville MS & 48434 \\
\hline Palm Springs CA & 46854 \\
\hline Wausau, Rhinelander WI & 46799 \\
\hline Twin Falls ID & 46528 \\
\hline Binghamton NY & 46299 \\
\hline Wheeling WV, Steubenville $\mathrm{OH}$ & 45822 \\
\hline Charlottesville VA & 45593 \\
\hline Ottumwa IA, Kirksville MO & 42315 \\
\hline Mankato MN & 40411 \\
\hline Meridian MS & 40196 \\
\hline Lima $\mathrm{OH}$ & 38265 \\
\hline Panama City FL & 37681 \\
\hline Bluefield, Beckley, Oak Hill WV & 35861 \\
\hline Bangor ME & 32568 \\
\hline Salisbury MD & 32563 \\
\hline Fairbanks AK & 32469 \\
\hline Juneau AK & 32406 \\
\hline Clarksburg, Weston WV & 31230 \\
\hline Parkersburg WV & 30981 \\
\hline Helena MT & 29943 \\
\hline Alpena MI & 28988 \\
\hline Elmira NY & 28647 \\
\hline Watertown NY & 27590 \\
\hline Eureka CA & 26925 \\
\hline Zanesville OH & 25372 \\
\hline Traverse City, Cadillac MI & 25187 \\
\hline North Platte NE & 24327 \\
\hline Marquette MI & 21441 \\
\hline Presque Isle ME & 9317 \\
\hline
\end{tabular}

\section{References}

Auchincloss, A. H. \& Roux, A. V. D. (2008). A new tool for epidemiology: The usefulness of dynamic-agent models in understanding place effects on health. American Journal of Epidemiology, 168(1), 1-8

Bloomfield, P. (2004). Fourier analysis of time series: An introduction. Chichester: John Wiley \& Sons

Brookmeyer, R., Boren, D., Baral, S. D., Bekker, L.-G., Phaswana-Mafuya, N., Beyrer, C. \& Sullivan, P. S. (2014). Combination HIV prevention among MSM in South Africa: Results from agent-based modeling. PloS one, 9(11), e112668

Casanova-Rosado, A. J., Medina-Solís, C. E., Casanova-Rosado, J. F., Vallejos-Sánchez, A. A., Minaya-Sánchez, M., Mendoza-Rodríguez, M., Márquez-Rodríguez, S. \& Maupomé, G. (2014). Tooth brushing frequency in Mexican schoolchildren and associated socio-demographic, socioeconomic, and dental variables. Medical Science Monitor, 20, 938 
Catteau, C., Blaizot, A., Duhamel, A., Delzenne, A., Devillers, A. \& Frimat, P. (2012). Dental status and related factors in an occupational health service in Northern France. Santé Publique, 25(6), 747-755

Catteau, C., Blaizot, A., Duhamel, A., Delzenne, A., Devillers, A. \& Frimat, P. (2014). Influence of the usual motivation for dental attendance on dental status and oral health-related quality of life. Medicina Oral, Patologia Oral y Cirugia Bucal, 19(3), e225

Day, T. E., Ravi, N., Xian, H. \& Brugh, A. (2013). An agent-based modeling template for a cohort of veterans with diabetic retinopathy. PloS one, 8(6), e66812

Frame, P. S., Sawai, R., Bowen, W. H. \& Meyerowitz, C. (2000). Preventive dentistry: Practitioners' recommendations for low-risk patients compared with scientific evidence and practice guidelines. American Journal of Preventive Medicine, 18(2), 159-162

Gilbert, G. H., Duncan, R. P. \& Kulley, A. M. (1997). Validity of self-reported tooth counts during a telephone screening interview. Journal of Public Health Dentistry, 57(3), 176-180

Gorman, D. M., Mezic, J., Mezic, I. \& Gruenewald, P. J. (2006). Agent-based modeling of drinking behavior: a preliminary model and potential applications to theory and practice. American Journal of Public Health, 96(11), 2055-2060

Hawley, G., Holloway, P. \& Davies, R. (1997). Dental health status associated with documented dental attendance patterns in adolescents. Community Dental Health, 14(1), 22-24

Laskowski, M., Demianyk, B. C., Witt, J., Mukhi, S. N., Friesen, M. R. \& McLeod, R. D. (2011). Agent-based modeling of the spread of influenza-like illness in an emergency department: A simulation study. IEEE Transactions on Information Technology in Biomedicine, 15(6), 877-889

Leischow, S. J. \& Milstein, B. (2006). Systems thinking and modeling for public health practice. American Journal of Public Health, 96(3), 403-405

Marshall, S., Northridge, M. E., De La Cruz, L. D., Vaughan, R. D., O’Neil-Dunne, J. \& Lamster, I. B. (2009). ElderSmile: A comprehensive approach to improving oral health for seniors. American Journal of Public Health, 99(4), 595-599

Mc Grath, C. \& Bedi, R. (2001). Public dental health: Can dental attendance improve quality of life? British Dental Journal, 190(5), 262-265

Metcalf, S. S., Northridge, M. E., Widener, M. J., Chakraborty, B., Marshall, S. E. \& Lamster, I. B. (2013). Modeling social dimensions of oral health among older adults in urban environments. Health Education \& Behavior, 40(1 suppl), 63S-73S

Newman, M. E. (2010). Networks: An introduction. Oxford: Oxford University Press

Peltzer, K. \& Pengpid, S. (2014). Oral health behaviour and social and health factors in university students from 26 low, middle and high income countries. International Journal of Environmental Research and Public Health, $11(12), 12247-12260$

Sheiham, A., Maizels, J., Cushing, A. \& Holmes, J. (1985). Dental attendance and dental status. Community Dentistry and Oral Epidemiology, 13(6), 304-309

Sohn, W. \& Ismail, A. I. (2005). Regular dental visits and dental anxiety in an adult dentate population. The Journal of the American Dental Association, 136(1), 58-66

Vehkalahti, M. \& Paunio, I. (1988). Occurrence of root caries in relation to dental health behavior. Journal of Dental Research, 67(6), 911-914

Watts, D. J. \& Strogatz, S. H. (1998). Collective dynamics of "small-world" networks. Nature, 393(6684), 440-442

Wiener, R. C. \& Shockey, A. T. (2014). Oral health knowledge and dental visits in community living older adults in rural Appalachia-West Virginia: A cross-sectional analysis. Journal of Studies in Social Sciences, 8(1), 1 